\title{
Spray Pyrolysed Nanostructured Gold-Doped Tin Oxide (Auto) Thin Films
}

\section{${ }^{* 1,3}$ AKINSOLA, SI; ${ }^{2}$ ADEDAYO, KS; ${ }^{3}$ ALABI, AB; ${ }^{4}$ OLANREWAJU, DB; ${ }^{5}$ AJAYI, AA; ${ }^{6}$ BABALOLA, OA}

\author{
${ }^{*}$ Department of Physical and Mathematical Sciences, Crown-Hill University, Ilorin, Nigeria \\ ${ }^{2}$ Department of physics, University of Maiduguri, Maiduguri, Nigeria \\ ${ }^{3}$ Department of Physics, University of Ilorin, Ilorin, Nigeria \\ ${ }^{4}$ Department of Physical Sciences, Landmark University, Oти-Aran, Nigeria \\ ${ }^{5}$ Department of Mathematical and Physical Sciences, Afe Babalola University, Ado-Ekiti, Nigeria \\ ${ }^{6}$ Sheda Science and Technology complex, Abuja, Nigeria \\ ${ }^{*}$ Corresponding Author Email: siakinsola711@gmail.com; Tel: +2348166602544; +2348024208631
}

\begin{abstract}
Nanostructured $\mathrm{SnO}_{2}$ thin films were grown by the chemical spray pyrolysis (CSP) method. Homemade spray pyrolysis technique is employed to prepare thin films. $\mathrm{SnO}_{2}$ is wide band gap semiconductor material whose film is deposited on glass substrate. A gold nanoparticle-doped tin oxide thin film (AuTO) was also prepared. UV-VIS (ultraviolet visible) spectroscopy and four-point probe analysis are done for optical and electrical analysis. UV-Visible absorption spectra show that the band gap of $\mathrm{SnO}_{2}$ thin film is $3.78 \mathrm{eV}$ and $3.82 \mathrm{eV}$ for AuTO. Band gap of $\mathrm{SnO}_{2}$ thin film can be tuned that it can be used in optical devices. The films have transmittance increases (to about 60\%) and the absorbance decreases in the visible region of the electromagnetic spectrum. The electrical conductivity of the Tin Oxide is enhanced by functionalizing with the Gold nanoparticles. It is higher than that of the Tin oxide only; $0.77 \times 10^{-2}(\mathrm{Ohm} \mathrm{cm})^{-1}$ and $3.55 \times 10^{-2}(\mathrm{Ohm} \mathrm{cm})^{-1}$ for $\mathrm{SnO}_{2}$ and AuTO respectively. These properties reveal that Tin Oxide doped with gold can actually be a good material for a transparent conducting oxide to be used in photovoltaic fabrication and in electronics.
\end{abstract}

\section{DOI: https://dx.doi.org/10.4314/jasem.v25i4.12}

Copyright: Copyright $\odot 2021$ Akinsola et al. This is an open access article distributed under the Creative Commons Attribution License (CCL), which permits unrestricted use, distribution, and reproduction in any medium, provided the original work is properly cited.

Dates: Received: 14 February 2021; Revised: 26 March 2021; Accepted: 12 April 2021

Keywords: Tin oxide, Spray pyrolysis, gold, nanoparticle, Transparent conducting oxide (TCO)

Tin oxide is known as a wide band gap semiconductor (energy band gap $3.6 \mathrm{eV}$ ), and it has only the tin atom that occupies the center of a surrounding core composed of six oxygen atoms placed approximately at the corners of a quasiregular octahedron, according to Patil, et al., (2012). Hoang and Shankar, (2011) reported that a key advantage of tin oxide over other contemporary semiconductors like silicon or gallium arsenide is its chemical stability and being corrosion resistant. Un-doped tin oxide $\left(\mathrm{SnO}_{2}\right)$ has band gap $>3$ $\mathrm{eV})$ and exhibits high optical transparency ( $\mathrm{T} \geq 85 \%)$ and an n-type character due to oxygen vacancies. The electrical conductivity of the material can be largely enhanced by doping with foreign impurities (Ziad, et al., 2014).

The deficiency of oxygen in $\mathrm{SnO}_{2}$ makes it a conductive material. Thin films of $\mathrm{SnO}_{2}$ have widely been used as front electrodes in photovoltaic devices and flat-panel displays, smart windows and phototransistors (Bhavana, et al., 2013). The most favoured dopants used by several researchers are antimony which substitutes the tin cations or by fluorine via substituting the oxygen atom. Fluorine doped tin oxide (FTO) exhibits good visible transparency as a result of its wide band-gap, while retaining a low electrical resistivity due to the high carrier concentration caused by the oxygen vacancies and the substitutional fluorine dopant. A survey of literature reveals that there are many techniques employed for the deposition of tin oxide thin film (Sumanta, et al., 2012).

Few of the methods are; thermal evaporation, DC Sputtering, chemical bath, spin coating, spray pyrolysis. Spray pyrolysis technique has a lot of advantages over several other methods for preparing thin film. The technique has been used in this study. Spray pyrolysis is a processing research technique being considered to prepare thin and thick films, ceramic coatings, and powders. Unlike many other film deposition techniques, spray pyrolysis is a simple and relatively cost-effective method (especially when considering the cost of equipment). It is an extremely easy technique for preparing films of any composition. Spray pyrolysis does not require high-quality substrates or chemicals (Udayakumar, et al., 2013). This technique has been used for deposition of dense 
films, porous films, and for powder production. Multilayered films can also be easily prepared using this flexible technique. Spray pyrolysis is being used for several decades in the glass industry and in solar cell production. A lot of work has been done on doping tin oxide with different elements like fluorine, antimony, Indium etc. and their effects on both the optical and electrical properties of the produced material have been studied. Much work has not been reported on doping tin oxide with gold vis-à-vis the effects on the electrical and optical properties of thin films. Gold is known to have high conductivity, chemical stability and a good corrosion resistant.

In this work, tin oxide has been doped with gold nanoparticles (hence the nomenclature AuTO) and the obtained optical and electrical properties, especially as applicable for use in solar cell have been studied. The objective of this work is to study the effect of functionalizing $\mathrm{SnO}_{2}$ with gold nanoparticle on its application as a transparent conducting oxide, as alternative to the already existing FTO and ITO glass.

\section{MATERIALS AND METHODS}

Materials: Tin tetrachloride penta-hydrate $\left(\mathrm{SnCl}_{4} .5 \mathrm{H}_{2} \mathrm{O}\right)$ and deionized water were used for synthesis of tin oxide. Gold (III) Chloride trihydrate $\left(\mathrm{HAuCl}_{4}\right)$ was used in addition for the doping.

Methods: Tin oxide was synthesized using spray pyrolysis technique. $0.1 \mathrm{M}$ of Tin tetrachloride pentahydrate $\left(\mathrm{SnCl}_{4} .5 \mathrm{H}_{2} \mathrm{O}\right)$ was used. The precursor, having been mixed with deionized water of required volume, were stirred by the magnetic stirrer (ARGO LAB M2A). Micro-slide glasses were used as substrate for the thin films. The substrates were pre-cleaned with detergent and deionized water and also with ethylene glycol in an ultrasonic cleaner (VWR Ultrasonic cleaner). The substrate temperatures were varied between $350^{\circ} \mathrm{C}$ and $450^{\circ} \mathrm{C}$, at the interval of $50^{\circ} \mathrm{C}$.

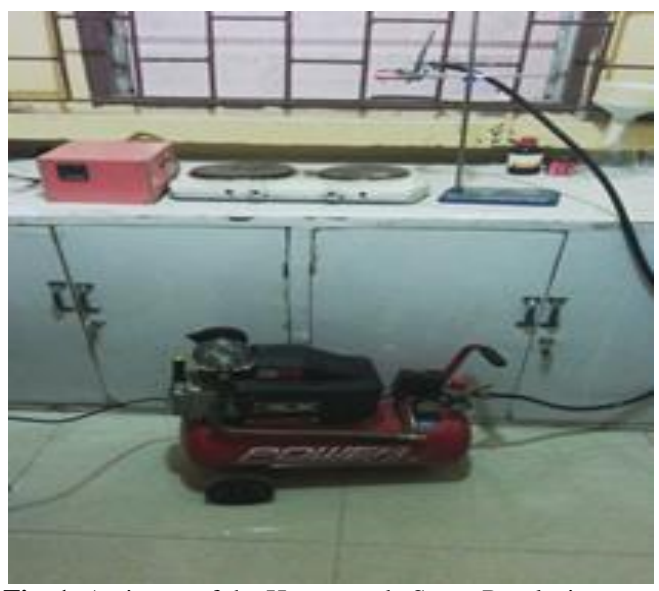

Fig. 1: A picture of the Home-made Spray Pyrolysis set up
The air pressure used was 2 bars and the distance between the nozzle and substrate was kept at $30 \mathrm{~cm}$. The solution was sprayed at a rate of $4 \mathrm{mlmin}^{-1}$. The films were annealed in a furnace at $500{ }^{\circ} \mathrm{C}$ for 1 hour. The sample with tin oxide only is named TO while the one with functionalized with gold is termed AuTO.

Characterization: The spectral for the transmittance and absorbance measurements were obtained using UV-VIS spectrophotometer in the wavelength range of $300 \mathrm{~nm}$ to $800 \mathrm{~nm}$. The $\mathrm{hf}(\mathrm{eV})$ value obtained by extrapolating the linear portion of the exponential curve from the graph of ( $\alpha \mathrm{hf})^{2}$ versus photon energy (hf) is the band gap energy of the material. The electrical property of the thin film was carried out using a Four-point Probe method. The Keithley Series Source meter was used for the sheet resistance $\left(R_{s}\right)$ of the thin film layer, and the obtained results were employed for estimating the electrical conductivity.

$$
\text { Resistivity }=\text { Rs } x \text { Film thickness }
$$

\section{RESULTS AND DISCUSSION}

Electrical properties: The conductivity is observed to be really enhanced by the addition of gold nanoparticles as seen in Table 1 . Gold is known to have high conductivity, chemical stability and a good corrosion resistant and hence a potential good dopant for improving the stability and conductivity of a metal oxide as a transparent conducting oxide (TCO) to be applied in optoelectronics devices and photovoltaic devices.

Table 1: Comparison of some electrical parameters of the Tin oxide and gold-doped thin oxide thin film

\begin{tabular}{lll}
\hline Samples & $\begin{array}{l}\text { Resistivity } \\
(\boldsymbol{\Omega} \mathbf{~ c m})\end{array}$ & $\begin{array}{l}\text { Conductivity } \\
(\boldsymbol{\Omega} \mathbf{~ c m})^{-1}\end{array}$ \\
\hline T.O & 129.05 & $0.77 \times 10^{-2}$ \\
AuTO & 28.15 & $3.55 \times 10^{-2}$ \\
\hline
\end{tabular}

Table 2: Comparison of the resistivity and conductivity of Tin

\begin{tabular}{lll}
\multicolumn{3}{c}{ oxide thin film at different substrate temperatures } \\
\hline $\begin{array}{l}\text { T.O (Substrate } \\
\text { Temperature) }\end{array}$ & $\begin{array}{l}\text { Resistivity } \\
(\boldsymbol{\Omega} \mathbf{~ c m})\end{array}$ & $\begin{array}{l}\text { Conductivity } \\
(\boldsymbol{\Omega} \mathbf{~ c m})^{-1}\end{array}$ \\
\hline $350^{\circ} \mathrm{C}$ & 28.31 & $3.53 \times 10^{-2}$ \\
$400^{\circ} \mathrm{C}$ & 1359.41 & $0.07 \times 10^{-2}$ \\
$450^{\circ} \mathrm{C}$ & 378.28 & $0.2 \times 10^{-2}$ \\
\hline
\end{tabular}

The I-V characteristics of the thin films show that it does not behave like an ohmic conductor, that is, the relationship of the current with voltage is not linear, hence a non-ohmic behaviour which typifies that of a semiconductor is observed and confirmed. The tin oxide prepared is confirmed to be a semiconductor from the I-V behaviour shown in the Fig.4. The high sheet resistance may be as a result of the relatively high thickness of the films. 

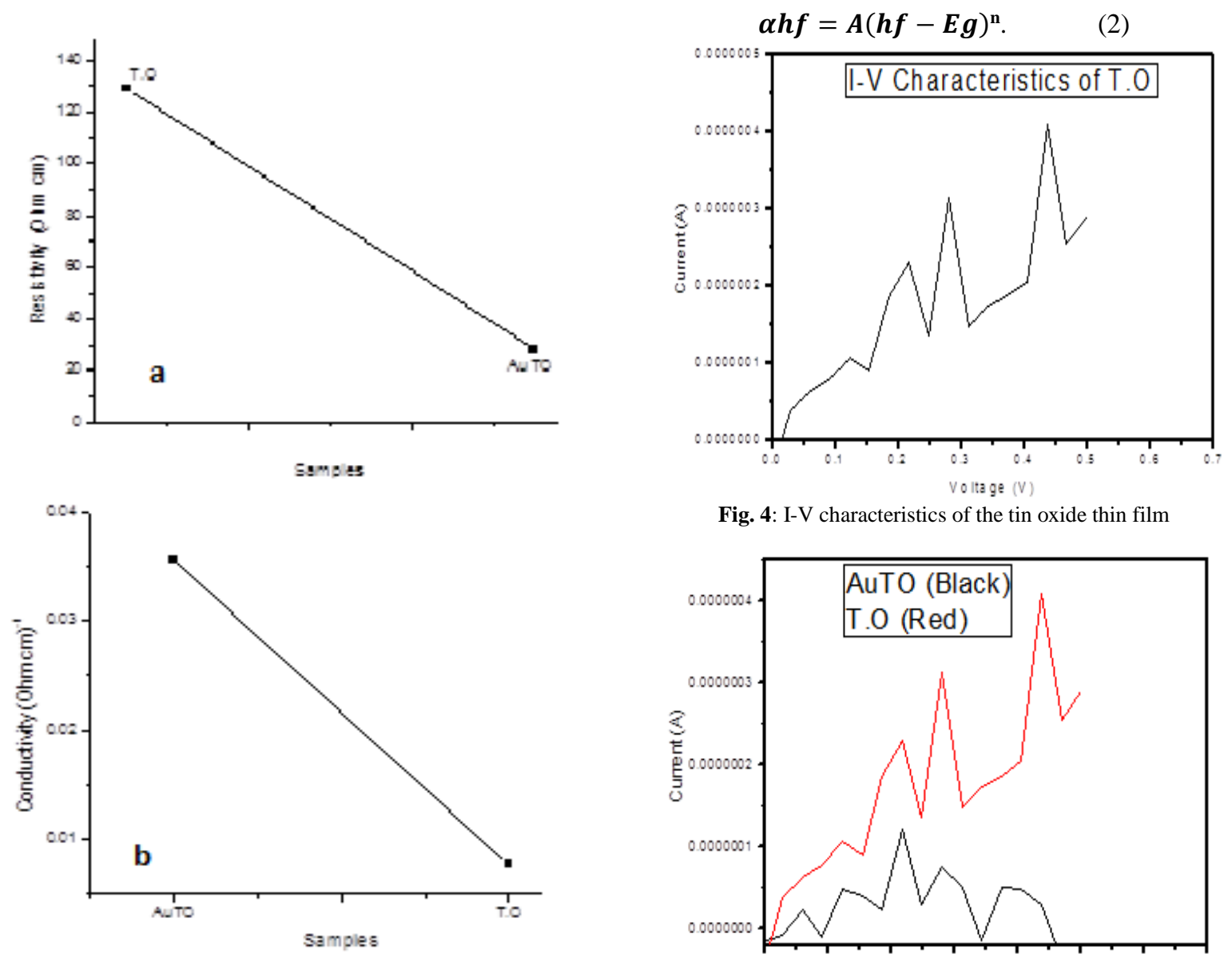

Fig. 4: I-V characteristics of the tin oxide thin film

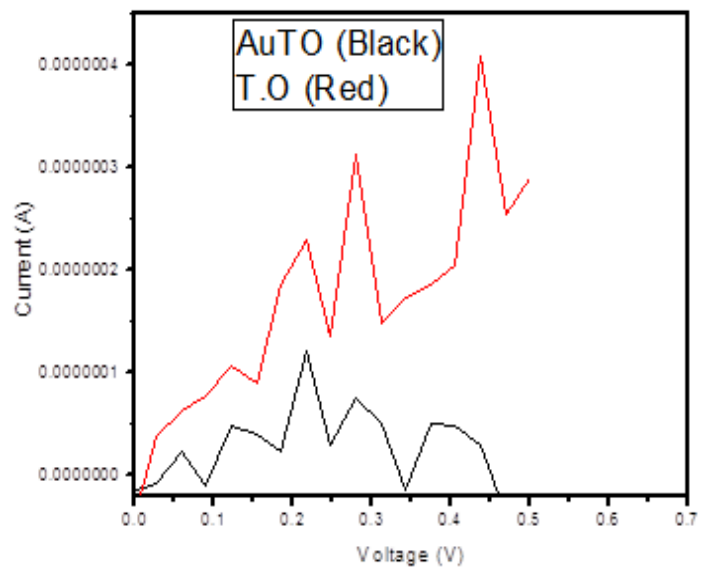

Fig. 5: Comparison of the I-V characteristics of the Gold -doped tin oxide and tin oxide only thin film

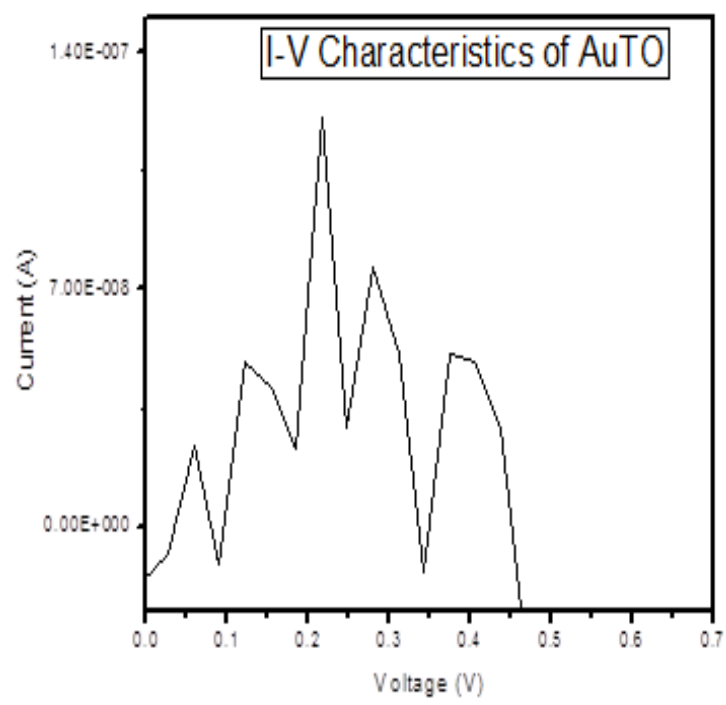

Fig. 3: I-V characteristics of the Gold -doped tin oxide thin film

Optical Properties: Estimation of $\mathrm{SnO}_{2}$ Optical Energy Band Gap: The energy band gap of the prepared films was estimated using the Tauc's relation

With $\mathbf{n}$ as $1 / 2$ for a direct transition, since the semiconductor compound $-\mathrm{SnO}_{2}$ is having a direct band gap, $\alpha$ is the absorption coefficient, the energy band gap was obtained from the plot of $(\alpha \mathrm{hf})^{2}$ vs hf. The absorption coefficient

$$
\alpha=\frac{2.303 \mathrm{~A}}{t},
$$

' $\mathrm{t}$ ' is the film thickness. This was done by extrapolating the linear portion of the plots to obtain the direct band gap of the films. $\mathrm{SnO}_{2}$ has an estimated direct transition of $3.78 \mathrm{eV}$ while the $\mathrm{SnO}_{2}$ thin film doped with gold nanoparticles have its band gap to be $3.82 \mathrm{eV}$. The band gap of the thin film was found to increase with the addition of the foreign material. This is because the energy gap between the valence band and the lowest empty state in the conduction band increased due to the filling of low-lying energy levels in the conduction band that is caused by the increase in the carrier concentration (Burstein-Moss effect). 


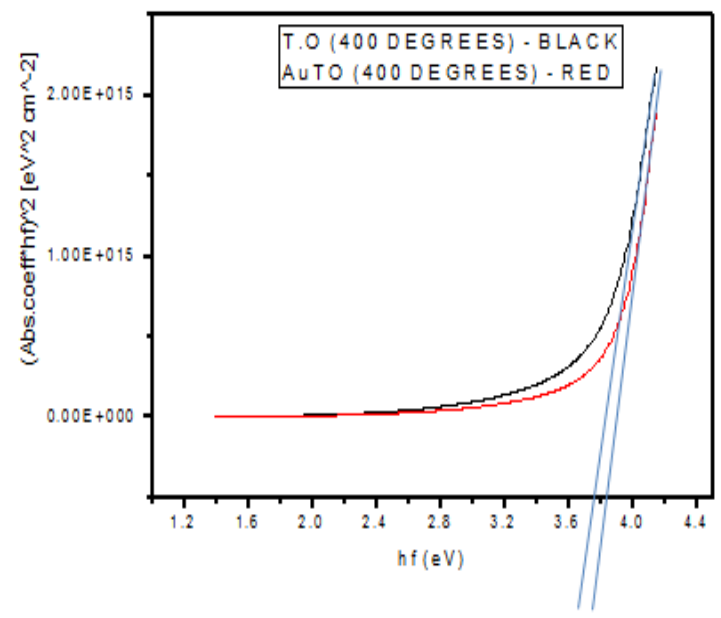

Fig.6: Energy band gap of both the un-doped Tin Oxide and the Gold-doped Tin Oxide (AuTO)

The shift in the band-gap can also be attributed to the variation in the average crystallite size, the internal stress or the free carrier concentration, according to Ziad, et al., 2014. The obtained value is close to the bulk value of the band gap of $\mathrm{SnO}_{2}$ and also in very close agreement with the report of Mishra et al., (2009). This wide band gap makes the material a good potential semiconductor in dye sensitized solar cell. Also, the band gap being tunable (even by functionalizing) suggests a suitable candidate for fabricating optoelectronic nanodevices. In semiconductor production, doping is the intentional introduction of impurities into an intrinsic semiconductor for the purpose of modulating its electrical properties. SnO has been reported as a remarkable n-type semiconductor by Suresh, 2015 among many researchers. Gold is a metal known as a transition metal, meaning that its number of valence electrons is not always constant, and will depend on the formula it is involved in.

However, gold most typically has one (1) valence electron, and occasionally three (3) valence electrons. The $\mathrm{HAuCl}_{4}$ used to functionalized tin oxide film prepared has gold with three valence electrons. N-type extrinsic semiconductor is produced by adding pentavalent atoms to a pure semiconductor while a $\mathrm{P}$ type semiconductor is obtained by the addition of trivalent atoms (with three valence electrons). In the ntype semiconductor there is the donor because of the excess electrons; the 'impurities' is the electron donor while that of a p-type is an electron acceptor. Tin can exhibit either 2 or 4 valence electrons and the addition of $\mathrm{HAuCl}_{4}$ makes the functionalized tin oxide a p-type semiconductor because, gold in the nanoparticle solution serves as an electron acceptor. Hence, a possibility of preparing a P-type transparent conductive oxide.
Transmittance and Absorbance spectra of $\mathrm{SnO}_{2}$ and AuTO: The thin film of Tin Oxide has an increasing transmission of electromagnetic spectrum within the visible region. The increase transmittance explains the reason for high optical transparency of $\mathrm{SnO}_{2}$ to Visible light (Ashok, et al., 2015), which makes it a good material for use as Transparent conducting Oxide (TCO) in fabricating solar cells and in Liquid crystals displays (LCD). The expected transparency is $>85 \%$. The range obtained in this study is between $50 \%$ and $60 \%$. This may be due to the high thickness and resistance of the thin film and probably a level of impurities presents in the synthesis, which is unavoidable, since a chemical method of deposition was employed, though to be improved upon by means to avoid these causes.

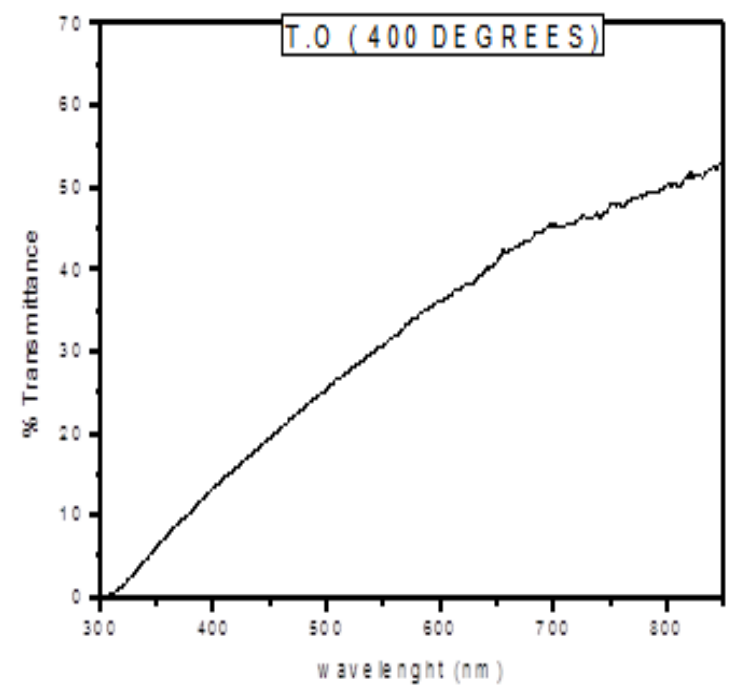

Fig.7: UV-Visible Transmission of $\mathrm{SnO}_{2}$ Thin film

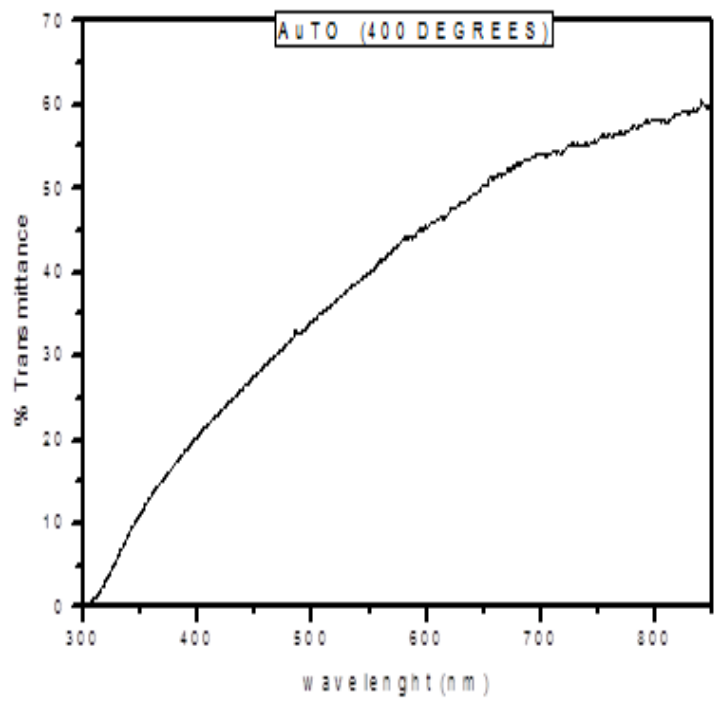

Fig.8: UV-Visible Transmission of Gold doped $\mathrm{SnO}_{2}$ Thin film 


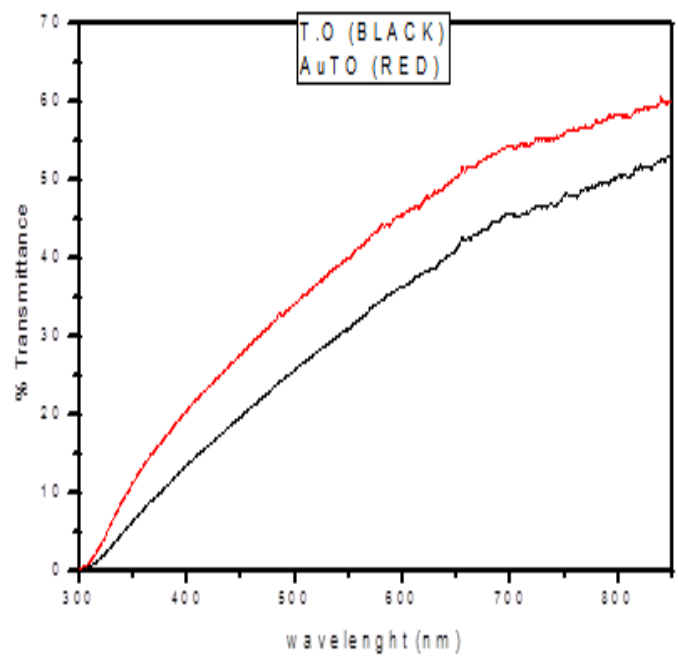

Fig. 9: Comparison of the Transmission of $\mathrm{SnO}_{2}$ and AuTO Thin film within the visible region

The variation in the transmission of visible light by the films prepared at different substrate temperature is shown in Fig. 10. The sample prepared at a temperature higher than the required temperature for the formation of $\mathrm{SnO}_{2}$ (which is around $400^{\circ} \mathrm{C}$ ) is seen to exhibit a very lower transmittance compared with ones below and at that temperature. This is a confirmation of the fact that substrate temperature required for the formation of Tin oxide is of importance in obtaining a $\mathrm{SnO}_{2}$ thin film.

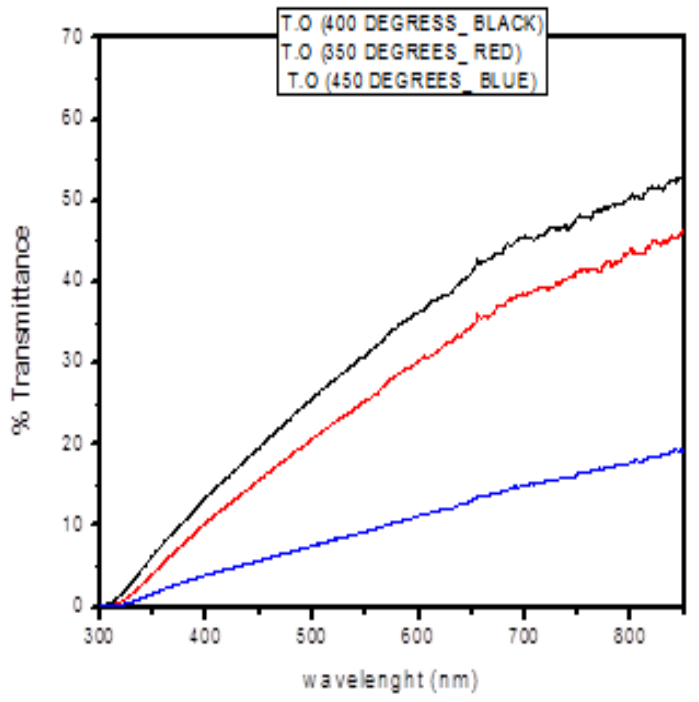

Fig. 10: Comparison of the Transmission of $\mathrm{SnO}_{2}$ Thin film deposited at different substrate temperature

The absorbance decreases within the visible region of the electromagnetic spectrum (Fig.11). This actually validated the fact that the material has a very low absorbance of UV-Visible light and a high transmittance of light in this region. The absorption peak of AuTo is around $289 \mathrm{~nm}$ while that of Tin oxide only is about $283 \mathrm{~nm}$. Also, the absorbance was reduced by the addition of the minute quantity of dopant, as seen in Fig. 11.

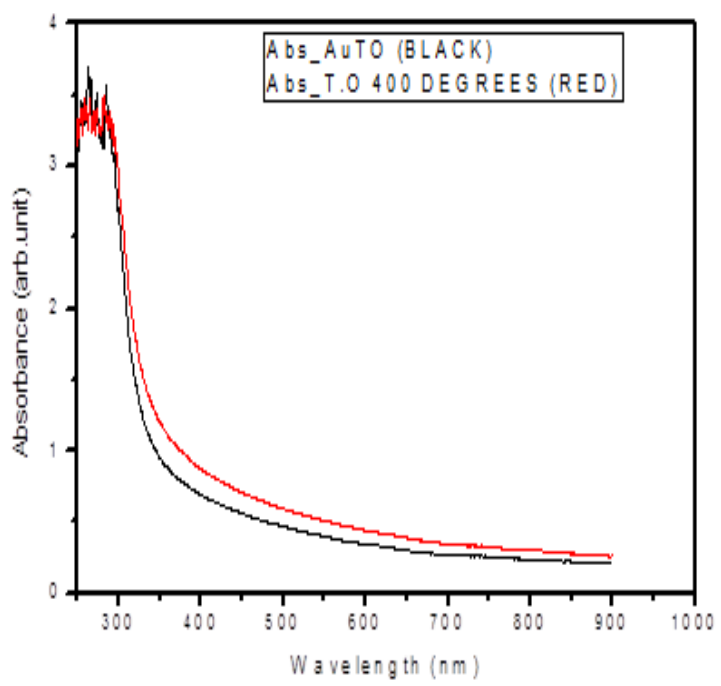

Fig. 11: Comparison of the Absorption of $\mathrm{SnO}_{2}$ and AuTO Thin film within the visible region

Conclusion: Thin film of tin oxide has been prepared on glass substrates. The effect of gold nanoparticles used to dope tin oxide was observed on both the electrical and optical properties of the thin film. A nomenclature AuTO for the gold doped tin oxide thin film is suggested, just as there is ITO and FTO for the Indium doped and Fluorine doped tin oxide respectively. The transparency of the prepared thin films increased while the conductivity is enhanced by the dopant (gold nanoparticles) introduced.

\section{REFERENCES}

Ashok, DB; Sachin SS; Balaprasad GA; Chandrashekhar MM. (2015). Synthesis of Nanostructured Tin Oxide ( $\mathrm{SnO} 2)$ Powders and Thin Films by Sol-Gel Method. J. Nano- Electron. Phys. 7(4) 0437: 1-4

Bhavana, NJ; Hyun, Y; Sam, SY. (2013). Structural, Optical and electrical properties of tin oxide thin films by electrostatic spray deposition. $J$. Electrostat. Elsevier. 71:48-52

Hoang,T and Shankar, BB. (2011). Synthesis and characterization of $\mathrm{N}$-and P-doped Tin Oxide Nanowires. Chemistry Faculty Publications and Presentation, Portland State University. 8-2011

Mishra, RL; Mishra, SK; Prakash, SG. (2009). Optical and Gas sensing characteristics of Tin Oxide Nanocrystalline thin film. J. Ovonic. Res. 5(4):77-85 
Sumanta, KT; Rajeswari, PV; and Bhabani, PH (2012). Synthesis of Tin Oxide Thin film and effect of Number of coating on Transmittance and Film Thickness. The African Review of Physics. 7 (0028)

Suresh Sagadevan (2015). Preparation, Structural and Electrical properties of Tin Oxide Nanoparticles. $J$. Nanomater. Mol. Nanotechnol. 4:1

Patil, GE; Kajale, DD; Gaikwad, VB; Jain, G.H. (2012). Spray pyrolysis deposition of
Nanostructured Tin Oxide Thin films. ISRN Nanomater.

Udayakumar, R; Khanaa, V; Saravanan, T. (2013). Synthesis and structural characterization of thin films of $\mathrm{SnO}_{2}$ prepared $\mathrm{BY}$ spray pyrolysis Technique. INDJSRT. 6:4754-4757

Ziad, YB; Peter, JK; Glen, W; Jeffery, B. (2014). Electrical and Optical properties of fluorine doped Tin Oxide Thin films prepared by Magnetron sputtering. Coat. 4:732-746 